\title{
LANDSCHAFTLICHE WANDLUNGEN IM NÖRDLICHEN CHURER RHEINTAL
}

\author{
WERNER NIGG
}

\begin{abstract}
Das Rheintal zwischen Reichenau und Sargans bildet in natur- und kulturgeographischer Hinsicht weitgehend eine Einheit. Es ist im folgenden versucht, den neueren Wandlungen im Talabschnitt nördlich von Chur nachzugehen.
\end{abstract}

\section{Natürliche Grundlagen}

Die rund $20 \mathrm{~km}$ lange Talschaft verläuft in süd-nördlicher Richtung und bildet eine breite, tiefe Bresche in der nordbündnerischen Bergwelt. Die Talsohle mit den weit ausladenden Schuttfächern ist im Mittel $3 \mathrm{~km}$ breit. Das Rheinbett liegt bei Chur 560, bei Fläsch $500 \mathrm{~m}$ ü. $\mathrm{M}$.

Die beiden Talhänge sind verschiedenartig. Die Ostflanke besteht zwischen den Einmündungen des Schanfigg und des Prätigau aus der Hochwang-Kette, die Höhen bis $1900 \mathrm{~m}$ erreicht und aus weichem penninischen Bündner Schiefer aufgebaut ist. Nördlich der Einmündung des Prätigau, der sogenannten Klus, folgt die Vilan-Falknis-Gruppe mit Gipfelhöhen bis $2562 \mathrm{~m}$. Sie wird zur Hauptsache aus weichem FlyschSchiefer aufgebaut; die schroffe Gipfelpartie des Falknis jedoch aus ostalpinen Kalken.

Die westliche Talflanke wird von der Kette Calanda (2706 m) - Pizalun (1478 m) gebildet. Hier sieht man deutlich die dicken helvetischen Kalkschichten ostwärts unter die Talsohle einfallen. Ebenfalls zu den helvetischen Decken gehört der relativ niedrige Fläscherberg, der, isoliert im Tal stehend, die nördliche Begrenzung des Bündner Rheintales markiert.

Die petrographischen Verhältnisse sind wesentlich mitverantwortlich an der Verschiedenartigkeit der beiden Talhänge. Die aus weichen Gesteinen bestehenden östlichen Bergzüge sind durch die Arbeit zahlreicher Rüfen (Wildbäche) stark zerfurcht worden und fallen zum Teil steil, fast senkrecht ab. Eine Reihe mächtiger Schuttfächer schiebt sich westwärts ins Tal vor und drängt den Rhein auf die Gegenseite. Ganz anders ist der Aufbau der Calandakette, deren steile Hänge durch das Einfallen der Kalkschichten gleichmäßiger sind als die der Ostflanke. Der widerstandsfähigere Kalk und wohl auch geringere und weniger heftige Regenfälle ließen kaum nennenswerte Seitentäler entstehen. Eine Ausnahme macht das Val Cosenz bei Untervaz; dort findet man denn auch den einzigen größeren Schuttfächer auf der Westseite.

Das Klima des Churer Rheintales ist für schweizerische Verhältnisse relativ trocken (1041 mm Jahresniederschläge beim Plantahof bei Landquart). Der niederschlagsreichste Monat ist der August $(128 \mathrm{~mm})$, der niederschlagsärmste der Februar $(52 \mathrm{~mm})$. Im Winter fällt mitunter viel Schnee, der jedoch zufolge häufiger Föhneinbrüche meistens rasch abschmilzt.

Der Föhn ist hier ein wesentliches Landschaftselement. Er tritt mitunter als heftiger, gefährlicher Sturm auf, er zaubert aber auch schöne, klare Oktobertage hervor, er verlängert den Herbst, verkürzt den Winter und hilft mit, daß unser Gebiet zu den nebelarmen Zonen der Schweiz gehört; werden doch im Mittel nur 8,4 Nebeltage gezählt (Zürich 43,5). Die durchschnittliche Jahrestemperatur beträgt beim Plantahof $7,6^{\circ}$, das Jahresmittel $-1,8^{\circ}$ und das Julimittel $16,6^{\circ}$. Im Churer Rheintal weht fast ständig ein Wind, am Tag der Talwind, während der Nacht der Bergwind. Setzen diese aus, dann folgt gewöhnlich der Föhn. Es gibt in diesem kleinen Gebiet bemerkenswerte Lokalklimate. So sind die östlichen Abhänge der Schuttfächer bedeutend wärmer als die gegenüberliegende Talseite. Besonders die Bündner Herrschaft, also der Kreis Maienfeld, die auch noch weitgehend gegen Norden geschützt ist, ist berühmt 
wegen des milden Klimas. Dort blühen die Obstbäume in den meisten Jahren zwei Wochen früher als in vielen Regionen des Mittellandes.

\section{Der Rhein}

Bis weit ins Mittelalter hinein waren der Rhein und zum Teil auch die Seitenbäche fast unbeschränkte Gestalter und Beherrscher der Talsohle. Mit seinen starken Schwankungen in der Wasserführung (bei der Tardisbrücke bei Mastrils: absolutes Maximum $1620 \mathrm{~m}^{3}$ Wasser pro Sekunde; absolutes Minimum $28 \mathrm{~m}^{3}$ ) verursachte der Rhein immer wieder Überschwemmungen. Bei jedem größeren Hochwasser suchte er sich einen neuen Weg und hinterließ Altläufe. Er brachte riesige Geschiebemassen aus den Hinterrheintälern, erodierte seitlich die untersten Schuttfächerpartien weg, schüttete: Sand- und Kiesflächen auf und verwandelte andere Teile der Talsohle in Sümpfe. Die Flußbreite war sehr unterschiedlich und nahm ein verhältnismäßig großes Gebiet ein. Kirchgraber (7, S. 24) berechnete, daß noch zu Beginn des 18. Jahrhunderts «der Rheinlauf mit seinem Geäder» allein im Gebiete des Kreises Fünf Dörfer nahezu 4 km² Fläche eingenommen hatte. Auch die Rüfen brachen bei jedem stärkeren Regen aus ihren Tobeln hervor und überschütteten die Schuttfächer stets von neuem mit Geschiebemassen.

So bot die Talniederung noch vor etwa 200 Jahren auf weiten Strecken das Bild von Naturlandschaften mit verwilderten Flußläufen, Altwässern, Auenwäldern und mit Heiden, auf denen knorrige Föhren und Dornensträucher wuchsen.

\section{Der Mensch im Kampf mit dem Rhein}

Der Mensch hatte bis in die Neuzeit hinein in der Talsohle wenig zu suchen. Er war froh, wenn er auf den vor dem Rhein geschützten Schuttkegeln seine Siedlungen bauen und sein Kulturland bestellen konnte, und er hatte mehr als genug zu tun, um mit den Rüfen fertig zu werden. Die Talniederung wurde nur extensiv und lange nicht überall als Weideland und zur Holzgewinnung genutzt. Einzig einige höher gelegene Partien, die durch Barrenbildung der Überschwemmungen entstanden waren, wurden als Ackerland genutzt.

Sicher haben die Talbewohner schon im Mittelalter versucht, da und dort den wilden Rhein zu zähmen. Doch mit der Kolonisation der Rheinniederung begann man erst im 16. und 17. Jahrhundert. Abgesehen von den Schwierigkeiten technischer Natur, fehlte es damals vor allem an einer Gesamtplanung und an einem gemeinsamen Vorgehen. Ja es kam zwischen benachbarten Gemeinden hin und wieder sogar zu rücksichtslosen Handlungen, indem man bei Hochwasser versuchte, die Fluten ins Gebiet des «lieben Nachbarn» abzuleiten.

Erst 1834 hat der Mensch die Herrschaft über den Rhein errungen, als die Kantone Graubünden und St. Gallen beschlossen, eine einheitliche Korrektion auszuführen. In der Folge bauten die Bewohner der Anstößergemeinden in nahezu fünfzigjähriger Gratisarbeit, im sogenannten Gemeindewerk, starke Wuhren. Einige Steinbrüche am Calandahang lieferten die mächtigen Felsblöcke, die mit den damals noch einfachen technischen Hilfsmitteln zum Rhein transportiert und zum Bau der Dämme gebraucht wurden. Damit erhielt der Rhein sein definitives Bett, das später noch verschiedentlich ausgebessert und verstärkt wurde, und das bis heute allen Hochwassern standgehalten hat. Natürlich mußten auch die Seitenbäche korrigiert und eingedämmt werden; bei den größeren Rüfen baute man sogenannte Schuttfänge. Doch bis in die Gegenwart brechen die Wildbäche immer wieder aus, reißen Brücken weg und überschütten mit ihrem Geschiebe Kulturland, Straßen- und Bahngeleise.

Auch die Landquart, die durch ihren weiten, flachen Schuttfächer fließt und dem Rhein zustrebt, wurde in Dämme gelegt. Einmal, anläßlich des Hochwassers von 1910, 
ist es ihr allerdings gelungen, ihre Wuhren wegzureißen und beträchtlichen Schaden an Verkehrswegen und Kulturland anzurichten.

\section{Wandlungen durch die landwirtschaftliche Erschließung und Nutzung}

Wie bereits dargelegi, besiedelten und bewirtschafteten die Bewohner bis in die Neuzeit hauptsächlich die Schuttfächer. Ausnahmen bilden das hochgelegene Dörfchen Says am Berghang ob Trimmis, das weit verstreute Dorf Masîrils am untern Abhang des Pizalun, die Weiler Rofels und Bovel ob Maienfeld und die kleine Siedlung Guscha, die hoch über dem Tal am steilen Falknishang klebt. Diese Siedlungen wurden von Walsern gegründet.

Kirchgraber (7, S. 183) glaubt, daß die Ortschaften auf den Schuttfächern schon im frühen Altertum bestanden haben. Lange bevor sich die Bewohner in die vom Rhein beherrschten Talniederungen wagten, bewirtschafteten sie außer den Schuttfächern die flacheren Berghänge als Maiensäße und hochgelegene, teils außerhalb des Tales liegende Alpweiden (z. B. Maienfelder-Alp bei Arosa, Malanser-Alp im Taminatal).

Ursprünglich war die Feldgraswirtschaft die einzige Nutzungsweise. In der ersten urkundlich überlieferten Zeit kam der Ackerbau dazu, der sich jedoch auf kleine Parzellen, sogenannte Quadern, die in der Nähe der Siedlungen lagen, beschränkte. Bis ins 18. Jahrhundert waren Roggen und Gerste die bedeutendsten Ackerpflanzen. Daneben wurde Spelz, Weizen, Hirse, Buchweizen, Hanf und Flachs angebaut. Langsam verbreiteten sich dann auch die Kartoffeln und der Mais.

Eine tiefgreifende Wandlung erfuhr die Talsohle mit der Rheinkorrektion und der darauf folgenden planmäßigen landwirtschaftlichen Erschließung. Das Neuland, das der Rhein freigeben mußte, wurde je nach Bodenqualität genutzt. Das ebene Gelände, das durch Aufschüttung von Schlamm besonders fruchtbar ist, wurde von den Gemeinden in sogenannte «Löser» aufgeteilt und den Bürgern kostenlos zur Nutzung als Acker- und Gemüseland zur Verfügung gestellt. Die sandigen und kiesigen Areale, die zum Teil mit ihren Föhren und Berberitzensträuchern parkähnliches Aussehen hatten, nutzte man als gemeinschaftliche Weiden, sogenannte Allmendweiden. Die moorigen und sumpfigen Teile, darunter auch die alten Rheinmäander mit Schilfbeständen, überließ man den Bauern zur Gewinnung von Streugras. Eigneten sie sich nicht einmal da$z u$, dann teilte man sie in rechteckige Parzellen auf, faßte sie mit Dämmen ein, um sie durch Aufschwemmen in fruchtbares Land zu verwandeln. Dieses Kolmationsverfahren wird an einigen Stellen heute noch fortgeführt, und die rechteckigen Weiher bilden ein besonderes Landschaftselement des Churer Rheintales. Schließlich ließ man vor allem in der Nähe der Rheinwuhren breite Streifen von Auenwäldern - hauptsächlich Erlenbestände - stehen; ja man hat sogar im Zusammenhang mit der Kolmation verschiedenerorts Erlen zur Bodenverbesserung angepflanzt.

Seit der systematischen Kolonisation der Rheinebene, also seit der Mitte des letzten Jahrhunderts, wird in größerem Ausmaß auch Mais angepflanzt, und nach dem Ersten Weltkrieg begann man zudem mit dem Anbau von Tabak.

An den Hängen mit bevorzugter Spalierlage kultiviert man seit der Römerzeit die Rebe. In der Herrschaft wurden die Weinberge zum dominierenden Landschaftselement, so daß man dieses Gebiet gerne auch etwa «Weinberg Graubündens»nennt. An geschützten Lagen entstanden in den letzten Jahrzehnten Handelsgärtnereien und Tafelobstkulturen. Mit Hilfe großer Glasgewächshäuser produziert das Churer Rheintal, vorab Malans, die «Primeurs» Graubündens.

Im 18. Jahrhundert machte man in Malans sogar den Versuch mit dem Anpflanzen von Feigen, Maulbeerbäumen und Edelkastanien, der allerdings bald wieder aufgegeben wurde. Dies zeigt, daß man schon zu jener Zeit das milde Klima der Bündner Herrschaft erkannt und vielleicht auch überschätzt hat. 
Der nachfolgende Auszug aus der Eidgenössischen Anbaustatistik 1960 gibt Auskunft über die hauptsächlichsten Ackergewächse und ihre Verbreitung in den einzelnen Gemeinden des nördlichen Churer Rheintals.

Die wichtigsten Ackergewächse nach Gemeinden 1960 (in Aren)

\begin{tabular}{|c|c|c|c|c|c|c|c|c|c|c|c|}
\hline & 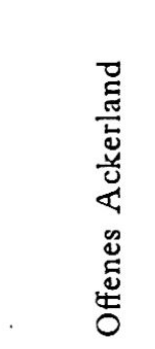 & 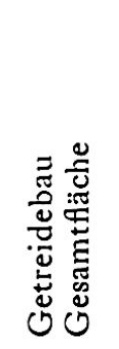 & 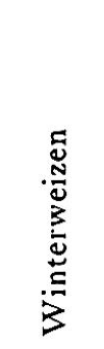 & 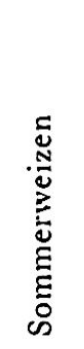 & 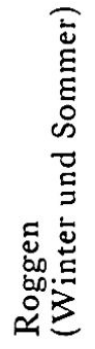 & 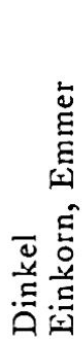 & 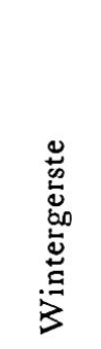 & 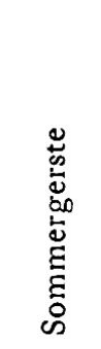 & 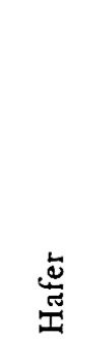 & 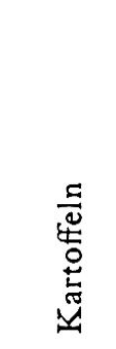 & 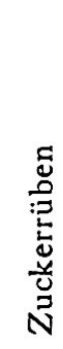 \\
\hline Kreis Fünf Dörfer & 44285 & 20612 & 9359 & 300 & 639 & 340 & 3586 & 1789 & 2859 & $12 \circ 22$ & 5 \\
\hline Haldenstein & 2318 & 883 & 384 & 8 & 45 & - & 208 & 101 & 100 & 1174 & 一 \\
\hline Igis & 14609 & 7951 & 4499 & 45 & 454 & 340 & 1322 & $26+$ & 415 & 2456 & 一 \\
\hline Mastrils & 585 & 79 & 40 & - & 30 & 一 & - & 9 & - & 398 & 一 \\
\hline Says & 677 & 277 & 105 & 14 & - & - & 21 & 67 & 67 & 369 & - \\
\hline Trimmis & 8088 & 4329 & 2174 & 10 & 84 & 一 & 588 & 368 & 772 & 2310 & 5 \\
\hline Untervaz & 7260 & $2 \circ 73$ & 463 & 62 & 8 & - & 255 & 557 & 349 & 2697 & 一 \\
\hline Zizers & 10748 & 5020 & 1694 & 161 & 18 & - & 1192 & 423 & 1156 & 2618 & 一 \\
\hline Kreis Maienfeld & 34434 & 18801 & 8458 & 598 & 316 & - & 3691 & 2351 & 2433 & 6767 & 一 \\
\hline Fläsch & 5786 & 3253 & 878 & 300 & - & 一 & 1012 & 67 & 592 & 1653 & 一 \\
\hline Jenins & 5068 & 2926 & 1649 & - & 60 & - & 99 & 507 & 448 & 1283 & 一 \\
\hline Maienfeld & 13820 & 7527 & 3485 & 65 & 238 & - & 1834 & 919 & 876 & 2656 & 一 \\
\hline Malans & 9760 & 5095 & 2437 & 233 & 18 & - & 746 & 858 & 517 & 1175 & \\
\hline
\end{tabular}

\begin{tabular}{|c|c|c|c|c|c|c|c|c|c|c|c|}
\hline & \multirow[b]{3}{*}{ 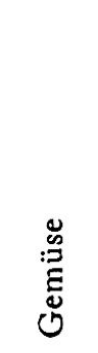 } & \multirow[b]{3}{*}{ 总 } & \multirow[b]{3}{*}{ 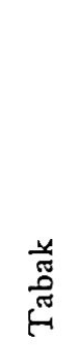 } & \multirow[b]{3}{*}{ 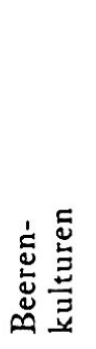 } & \multirow{3}{*}{ 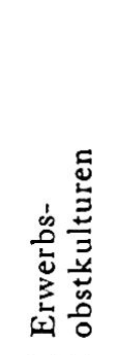 } & \multicolumn{5}{|c|}{ Rebland } & \multirow[b]{3}{*}{ 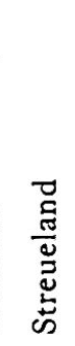 } \\
\hline & & & & & & \multirow{2}{*}{ 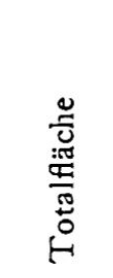 } & \multicolumn{2}{|c|}{$\begin{array}{c}\begin{array}{c}\text { Veredelte } \\
\text { Reben }\end{array} \\
\end{array}$} & \multicolumn{2}{|c|}{$\begin{array}{l}\text { Direkt- } \\
\text { träger }\end{array}$} & \\
\hline & & & & & & & 吾 & 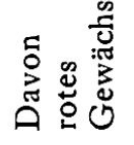 & $\stackrel{\pi}{0}$ & 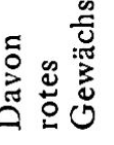 & \\
\hline Kreis Fünf Dörfer & 2286 & $27 \circ$ & 662 & $20+$ & 1121 & 1837 & 1811 & 1793 & 26 & 26 & 408 \\
\hline Haldenstein & 50 & - & - & - & 4 & - & - & - & - & - & 一 \\
\hline Igis & 1014 & $27 \circ$ & - & 31 & 91 & 323 & 303 & 298 & 20 & 20 & 一 \\
\hline Mastrils & 21 & - & 一 & - & - & - & - & - & - & - & 15 \\
\hline Says & 31 & - & - & - & - & - & - & - & - & - & 258 \\
\hline Trimmis & 439 & - & 20 & 12 & 30 & 548 & 542 & 529 & 6 & 6 & 29 \\
\hline Untervaz & 318 & 一 & 560 & 2 & - & - & - & - & - & 一 & - \\
\hline Zizers & 413 & - & 82 & 159 & 996 & 966 & 966 & 966 & - & - & 106 \\
\hline Kreis Maienfeld & 2260 & 150 & 103 & 109 & 1087 & 13747 & 13681 & 13577 & 45 & 42 & 489 \\
\hline Fläsch & 96 & - & - & - & 9 & 2060 & 2060 & 2060 & - & - & 71 \\
\hline Jenins & 165 & - & - & 1 & - & 2569 & 2524 & 2524 & 45 & 42 & 110 \\
\hline Maienfeld & 346 & 150 & 103 & 25 & 169 & 5444 & 5429 & 5423 & - & - & 292 \\
\hline Malans & 1653 & - & - & 83 & 909 & 3674 & 3668 & 3570 & - & - & 16 \\
\hline
\end{tabular}

Die letzte Wandlung, die sich im Zusammenhang mit der landwirtschaftlichen Bewirtschaftung zeigt, wird durch die Mechanisierung und die Güterzusammenlegungen verursacht. Auenwälder, alte Baumgruppen und Heckenbestände verschwinden immer mehr. Wo noch in den zwanziger Jahren idyllische Weiher, Schilfbestände und parkähnliche Landschaften den Wanderer erfreuten, dehnen sich heute weite, gut gepflegte Äcker, Baumgärten und Kunstwiesen mit zum Teil schnurgeraden Straßen aus. Die Erträge der Landwirtschaft sind wesentlich gesteigert, dafür aber schöne ursprüngliche Landschaften geopfert worden. Im Zusammenhang damit steht auch die bedauernswerte Verarmung der Fauna, besonders der Vogelwelt. 


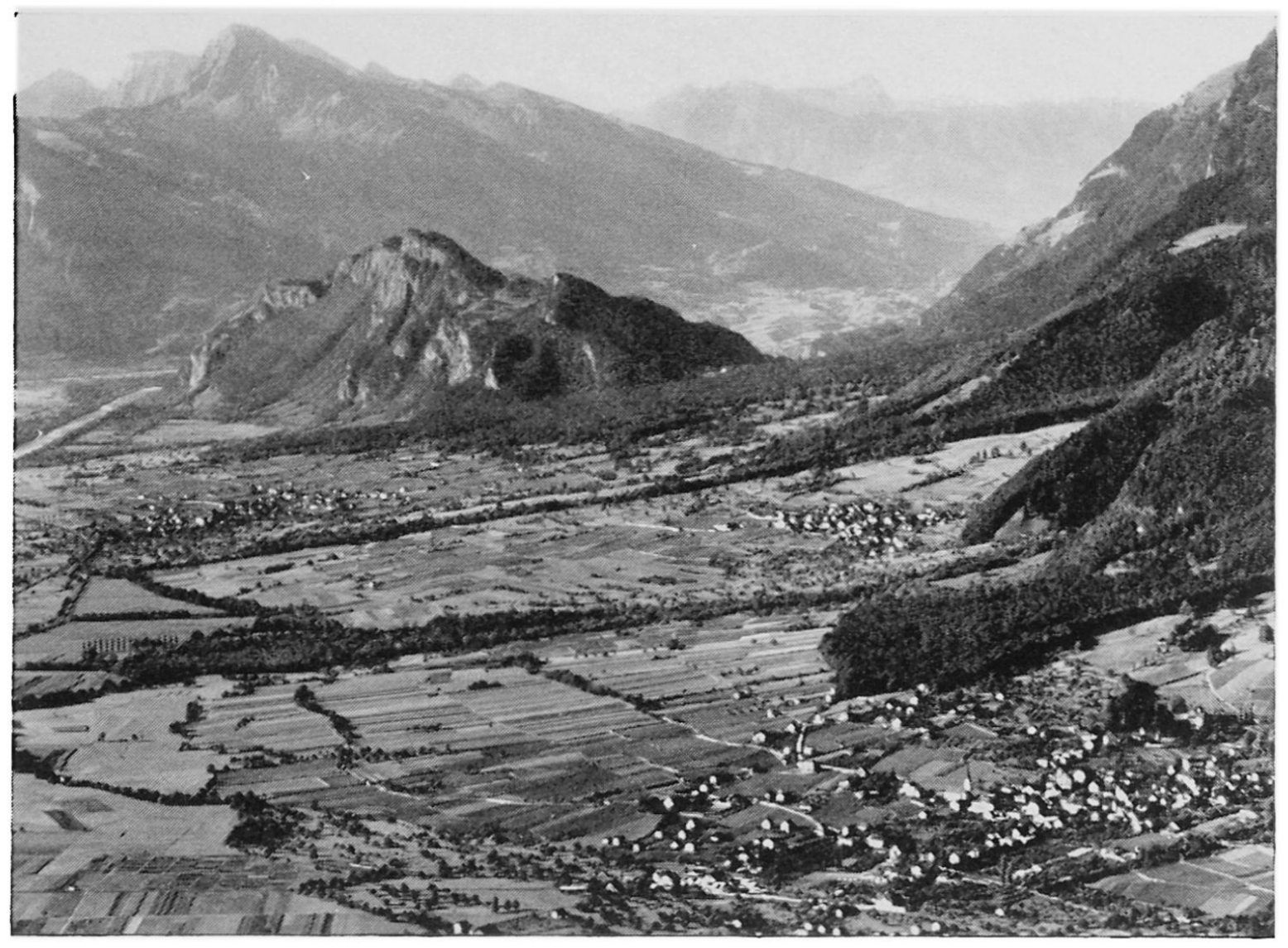

Abb. 1 Die Bündner Herrschaft. Auf den sanft ansteigenden Schuttfächern liegen (von links nach rechts) die Ortschaften Maienfeld, Jenins und Malans. Zwischen dem Fläscherberg (Mittelgrund) und dem Abhang des Falknis (rechts) erkennt man die breite Paßlücke St. Luzisteig. Links des Fläscherbergs sieht man ein kurzes Stück des korrigierten Rheinlaufs. Die langgezogenen Waldstreifen verlaufen den Rüfen (Wildbächen) entlang. (Photo: J. Geiger, Flims-Waldhaus.)

\section{Wandlungen durch den Verkehr}

Das Churer Rheintal wird etwa als «Korridor Graubündens»bezeichnet. Es ist jedoch nicht nur Eingang in das «Alpenland der 150 Täler», es ist ebensosehr Hauptzugang zu zahlreichen wichtigen Alpenpaßübergängen, von denen einige uralt sind. Zur Römerzeit traf in Magia (Maienfeld) die Straße von Brigantium (Bregenz), welche über die Luzisteig führte, mit der Straße von Turicum (Zürich) zusammen und führte von dort, die Rheinebene meidend, über die Schuttfächer von Siedlung zu Siedlung entlang der östlichen Talflanke nach Curia Raetorum (Chur). Die wichtige Verkehrslage war der Hauptgrund dafür, daß Maienfeld stark befestigt wurde und früh das Stadtrecht erhielt.

Bis zum Bau der «Oberen Zollbrugg» südlich von Malans über die Landquart (im 14. Jahrhundert) und der «Unteren Zollbrugg» über den Rhein bei Mastrils (1529), die damals die einzige Brücke zwischen Reichenau und Bodensee war, verlief die Straße nach Möglichkeit auf geschützten Anhöhen. Zur Sicherung des Verkehrsweges entstanden im Mittelalter und in der Neuzeit eine große $Z$ ahl von Burgen und Schlössern, von denen heute noch verschiedene erhalten sind, während andere nur noch als Ruinen von der Vergangenheit zeugen.

Die erste moderne Straße wurde 1782-1786 gebaut. Sie verlief von Maienfeld direkt zur Oberen Zollbrugg, dann über die östlichen Schuttfächer nach Chur, wobei sie ein- 
zig durch die Ortschaft Zizers führte. Zizers hat dadurch den Charakter eines Straßendorfes erhalten. An der neuen Straße wurden mehrere große Gasthäuser mit Stallungen gebaut, um Fuhrleuten, Reisenden und Pferden Unterkunft zu bieten.

Im Jahre 1858 wurde die Linie Rheineck-Chur der Vereinigten Schweizer Bahnen dem Betrieb übergeben. Die erste Station auf Bündner Boden erhielt das Städtchen Maienfeld, die zweite wurde in eine «verlassene Ödlandschaft» zwischen der Landquartmündung und dem Rhein gebaut. Bald entstand dort eine kleine Siedlung, «Landquart-Au» genannt. Dann baute man der Landquart entlang die Fahrstraße durch die Klus ins Prätigau. Bald entwickelte sich auf dieser Straße ein reger Verkehr. Postkutschen mit Gepäckwagen und private Fuhrwerke rollten Richtung Prätigau und Davos. In Landquart wurden zeitweise 150-200 Pferde untergebracht. Es entstanden Gasthäuser, ein Hotel, Ställe, eine Schmiedewerkstätte und 1868 eine Ziegelei. Damit war Landquart, die erste größere Ortschaft auf dem einst gefürchteten Talboden, gegründet. Allerdings gehört diese Siedlung auch heute noch politisch zur Gemeinde Igis. Südlich der Station entstand 1887 eine Holzschleiferei, aus der sich dann die ersten größeren Fabrikanlagen auf Bündner Boden - die Papierfabriken Landquart - entwickelten, denen bis 1917 auch eine Maschinenfabrik angegliedert war.

1889 wurde die Schmalspurbahnstrecke Landquart-Klosters, 1890 die Strecke Klosters-Davos und 1896 Landquart-Chur-Thusis dem Betrieb übergeben. Die Rhätische Bahn baute nun in Landquart das Hauptdepot, die Reparaturwerkstätte und ein Lagerhaus. Wie in Amerika während der Pionierzeit errichtete das Bahnunternehmen für seine zahlreichen Angestellten und Arbeiter eine ganze Wohnsiedlung mit schachbrettartigem Grundriß. Neben den Ein-, Zwei- und Vierfamilienhäusern, die alle gleich aussahen, baute die Bahn auch ein Schulhaus, ein Hotel, einen Kaufladen und das Postgebäude. Sogar für die Versorgung mit Wasser und elektrischer Energie war sie besorgt. So ist Landquart ein bedeutender Eisenbahnknotenpunkt geworden. 408 Personen finden heute in Landquart bei der Bahn Arbeit, nämlich

83 als Bahnhofangestellte

200 in der Werkstätte der Rhätischen Bahn (Rh. B.)

10 in der Materialverwaltung der Rh. B.

48 als Lokomotivführer der Rh. B.

67 andere Angestellte der Rh. B.

Dank der günstigen Verkehrslage hat sich nicht nur die Papierfabrik zu einem Großunternehmen mit 500 Angestellten und Arbeitern entwickelt, es sind in Landquart auch zahlreiche kleinere Industrie- und Handelsbetriebe entstanden. 1960 waren von den 1503 Berufstätigen der Gemeinde Igis-Landquart 1245 oder $76 \%$ in Industrie, Handwerk, Baugewerbe, Handel und Verkehr beschäftigt. Aus der einstigen kleinen Eisenbahnersiedlung ist das Wirtschaftszentrum des nördlichen Churer Rheintales mit modernen Geschäftsstraßen und Wohnblockquartieren entstanden.

Aber auch den andern Dörfern hat der Bahnbau Änderungen gebracht. Einige Ortschaften wie Maienfeld, Malans, Igis und Zizers haben sich gegen die Station hin ausgedehnt, so daß eigentliche Bahnhofquartiere entstanden.

Die letzte große Wandlung durch den Verkehr brachte der Bau der Nationalstraße (N 13), die keine einzige Siedlung tangiert. Das Teilstück Landquart-Trimmis wurde 1958 in Betrieb genommen. Seither ist die Straße von Heiligkreuz bei Sargans bis Chur befahrbar. Das breite Band der Straße, die verschiedenen Anschlußwerke und vor allem die großzügigen Straßen- und Bahnbrücken zwischen Zizers und Untervaz sind auffallende neue Landschaftselemente. Zweifellos wird auch dieser neue Verkehrsweg Anreiz zur Errichtung weiterer Bauten geben. 


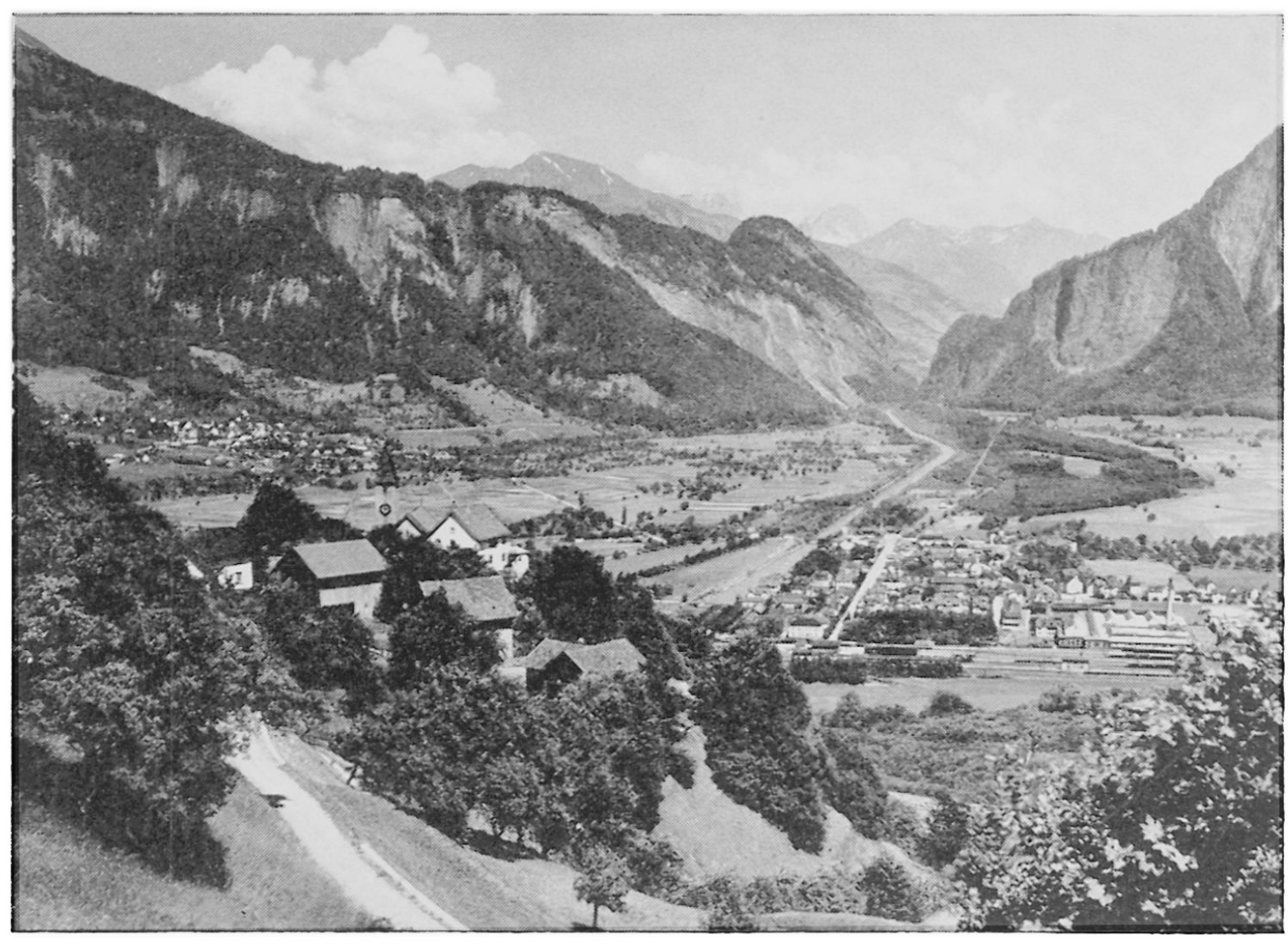

Abb. 2 Das Churer Rheintal bei Landquart. Im Vordergrund links die katholische Kirche und einige Häuser von Mastrils. Im Mittelgrund die Talebene mit dem korrigierten Lauf der Landquart und die gleichnamige Siedlung. Auf dem Schuttfächer links Malans. Im Hintergrund die Klus, der Eingang ins Prätigau und einige Gipfel der Rhätikonkette.

\section{Wandlungen durch die Industrie}

Die gute Verkehrslage und das flache Gelände sind günstige Voraussetzungen für den Bau von Industrien. Die amerikanisch anmutende Entwicklung von Igis-Landquart wurde bereits erwähnt. Eine neue Erscheinung im Landschaftsbild ist ein Zementwerk bei Untervaz, das 1958 den Betrieb eröffnet hat und rund 50 Personen beschäftigt. Am Rhein stehen einige Kieswerke, die den Kies direkt aus dem Rheinbett baggern. Kleinere Industrieunternehmen entstanden auch in Trimmis, so eine Zementwarenfabrik und eine Gießerei.

In der letzten Zeit war auch schon vom Projekt einer Oelraffinerie im Churer Rheintal die Rede, führt doch die Pipeline Genua-Ingoldstadt durch das Churer Rheintal. Es scheint, daß dieser Plan mit zahlreichen Gegnern zu rechnen hat, nicht zuletzt mit den Weinbauern der Herrschaft und dem Kurort Bad Ragaz.

Im Kreis Maienfeld ist die Lage zu den neuen Verkehrswegen ungünstiger als im Kreis Fünf Dörfer. Dort ist demzufolge die Industrie bis heute von untergeordneter Bedeutung. Bis in die Nachkriegszeit war eine Baumwollzwirnerei in Malans mit einer Belegschaft von 20-30 Personen das einzige nennenswerte Unternehmen. In den letzten Jahren bemüht sich Maienfeld, in der Nähe des Bahnhofs einige Fabrikbetriebe anzusiedeln. Trotzdem zeigt auch die Bündner Herrschaft eine neue Wandlung, denn immer mehr werden von Pendlern außerhalb der alten Siedlungen - zum Teil inmitten der Rebberge - moderne, oft fremd wirkende Einfamilienhäuser erstellt, was nicht zur Förderung der Harmonie der Landschaft beiträgt. 


\section{Bevölkerung}

Es wäre eine interessante und umfangreiche Arbeit, die Wandlungen der einzelnen Siedlungen und besonders die Veränderungen in der Struktur der Bevölkerung eingehend zu untersuchen. Wir müssen uns mit der allgemeinen Feststellung begnügen, daß sich die Gesamtzahl der Bewohner im Kreis Fünf Dörfer in den letzten hundert Jahren nahezu verdoppelt hat, währenddem sic im Kreis Maienfeld nur eine Zunahme von rund $17 \%$ erfuhr. Auch die Zahl der zugewanderten und der nicht in der Landwirtschaft beschäftigten Personen ist im Kreis Fünf Dörfer viel größer als in der Herrschaft. Es zeigt sich also deutlich, wie groß der günstige Einfluß der Lage an der groBen Durchgangsroute ist. Es ist aber auch mit Bestimmtheit anzunehmen, daß die ruhigere Entwicklung im Kreis Maienfeld zu einem guten Teil mit der Mentalität, besonders mit der Liebe zum angestammten Beruf, die bei den Weinbauern in der Herrschaft immer noch groß ist, zusammenhängt.

Die nachstehenden Zusammenstellungen aus der Eidgenössischen Statistik geben für jede Gemeinde Auskunft über die Entwicklung der Wohnbevölkerung von 1850 bis 1960, über Heimat, Konfession und Muttersprache sowie über die Aufteilung in verschiedene Berufsgruppen der Wohnbevölkerung im Jahre 1960.

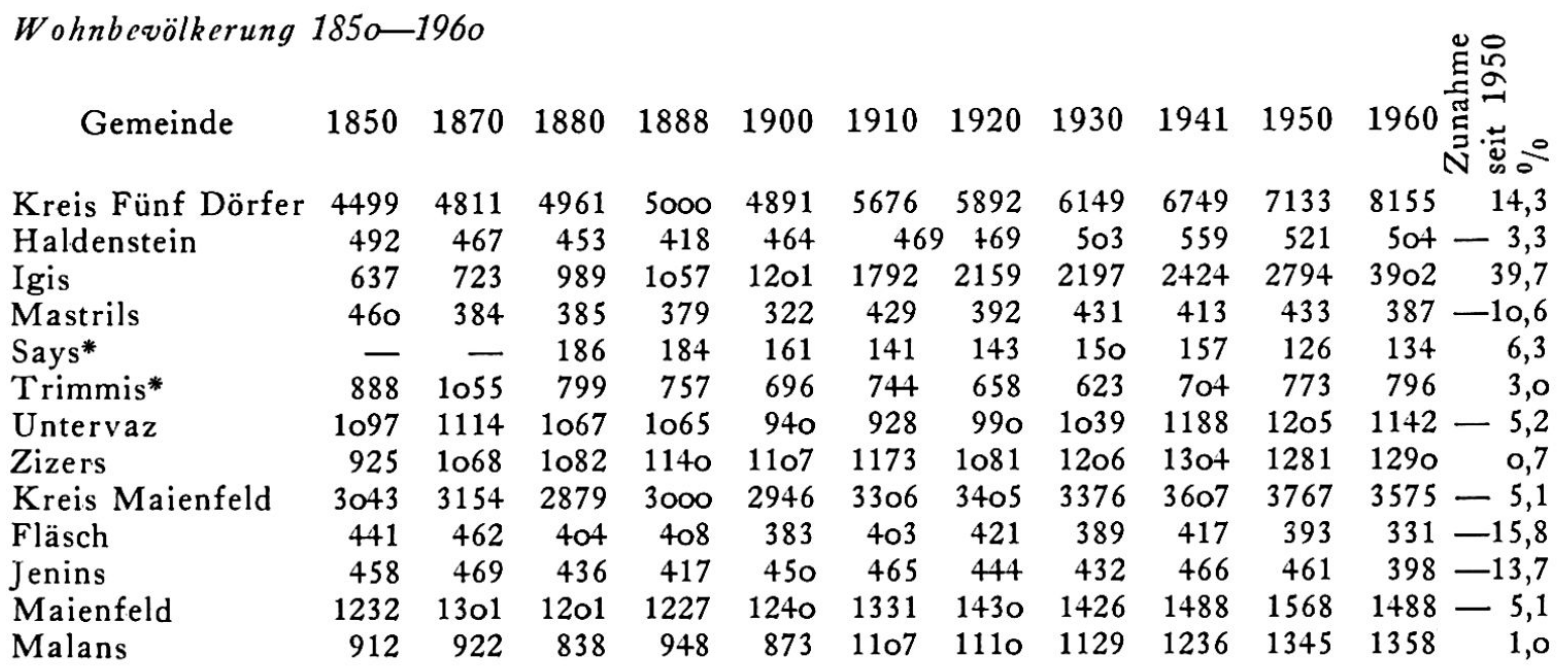

* Die Gemeinde Says wurde 1875 von der Gemeinde Trimmis abgetrennt.

\section{Ausblick}

Um 1800 hat der weitblickende Geograph und Agronom Carl Ulisse von Salis bereits die günstige Lage von Landquart erkannt und darüber geschrieben:

«Anstatt des von Verheerung umgebenen Zollhauses zaubere man sich in die Wüste zwischen Rhein und Landquart eine der schönsten, durch Bearbeitung gewonnene Gegend, geziert durch einen wohlgebauten Flecken, den Kaufleute, Fabrikanten und Landwirte beleben. Keine Lage in Bünden bietet mehr Vorteile zu einer solchen Ansiedlung dar als diese, und sie könnte das Mittel abgeben, eine totale Umwälzung in ökonomischen und industriösen Zuständen des Landes zuwege zu bringen.»

150 Jahre später ist seine Prophezeiung oder sein Wunschtraum Wirklichkeit geworden. Was C. U. von Salis über Landquart gesagt hat, könnte man heute mit einigen Abwandlungen vom ganzen nördlichen Churer Rheintal, besonders vom Kreis Fünf Dörfer, sagen: Kein anderes Gebiet Graubündens zeigt solche Vorteile für eine industrielle und wirtschaftliche Entwicklung wie dieses. Einige Anfänge sind schon gemacht, und es ist allerhöchste Zeit, daß sich verantwortungsbewußte Planer dieser Landschaft annehmen. Eine Planungskommission ist an der Arbeit (11, Seite 247). Hoffen wir, daß es ihr gelingen wird, das nördliche Churer Rheintal zu einem optimal schönen und gesunden Lebensraum für die zukünftigen Bewohner zu gestalten. 
Wohnbevölkerung 1960 nach Heimat, Konfession und Muttersprache

\begin{tabular}{|c|c|c|c|c|c|c|c|c|c|c|c|c|c|c|c|c|}
\hline & \multicolumn{6}{|c|}{ Heimat } & \multicolumn{5}{|c|}{ Konfession } & \multicolumn{5}{|c|}{ Muttersprache } \\
\hline & \multicolumn{2}{|c|}{ Wohngemeinde } & \multirow{2}{*}{\begin{tabular}{|c|} 
Andere \\
Gemeinden \\
des Wohn- \\
kantons
\end{tabular}} & \multirow{2}{*}{\begin{tabular}{|l|} 
Andere \\
Kantone
\end{tabular}} & \multicolumn{2}{|c|}{ Ausland } & \multirow[b]{2}{*}{\begin{tabular}{|c|}
$\begin{array}{c}\text { Prote- } \\
\text { stantisch }\end{array}$ \\
\end{tabular}} & \multirow{2}{*}{$\begin{array}{l}\text { Römisch- } \\
\text { katholisch }\end{array}$} & \multirow{2}{*}{$\begin{array}{c}\text { Christ- } \\
\text { katholisch }\end{array}$} & \multirow{2}{*}{$\begin{array}{c}\text { Israeli- } \\
\text { tisch }\end{array}$} & \multirow{2}{*}{\begin{tabular}{|c|} 
Andere \\
und ohne \\
Kon- \\
fession \\
\end{tabular}} & \multirow[b]{2}{*}{ Deutsch } & \multirow{2}{*}{$\begin{array}{l}\text { Franzö- } \\
\text { sisch }\end{array}$} & \multirow{2}{*}{$\begin{array}{c}\text { Italie- } \\
\text { nisch }\end{array}$} & \multirow{2}{*}{$\begin{array}{c}\text { Räto- } \\
\text { romanisch }\end{array}$} & \multirow[b]{2}{*}{ Andere } \\
\hline & $\begin{array}{c}\text { Im } \\
\text { ganzen }\end{array}$ & \begin{tabular}{|l|} 
Daselbst \\
geboren
\end{tabular} & & & Total & \begin{tabular}{|l|}
$\begin{array}{l}\text { Dav. Nie- } \\
\text { dergelass. }\end{array}$ \\
\end{tabular} & & & & & & & & & & \\
\hline Kreis Fünf Dörfer & 2484 & 1853 & 2816 & 2328 & 527 & 267 & 4298 & 3828 & 3 & 1 & 25 & 7289 & 33 & 330 & 487 & 16 \\
\hline Haldenstein & 226 & 174 & 165 & 75 & 38 & 4 & 426 & 77 & - & - & 1 & 432 & 4 & 29 & 34 & 5 \\
\hline Igis & 320 & 202 & 1717 & 1517 & 348 & 196 & 2344 & 1540 & 3 & 1 & 14 & 3292 & 21 & 232 & 347 & 10 \\
\hline Mastrils & 188 & 119 & 111 & 80 & 8 & 4 & 151 & 236 & - & - & - & 373 & 1 & 4 & 9 & - \\
\hline Says & 63 & 35 & 35 & 34 & 2 & - & 112 & 22 & - & - & - & 131 & 1 & - & 2 & - \\
\hline Trimmis & 332 & 260 & 258 & 171 & 35 & 14 & 358 & 433 & - & - & 5 & 749 & - & 29 & 17 & 1 \\
\hline Untervaz & 870 & 696 & 138 & 110 & 14 & 16 & 285 & 857 & - & - & - & 1106 & 1 & 11 & 24 & - \\
\hline Zizers & 485 & 367 & 392 & 341 & 72 & 33 & 622 & 663 & - & - & 5 & 1206 & 5 & 25 & 54 & - \\
\hline Kreis Maienfeld & 1496 & 1109 & 1000 & 802 & 277 & 105 & 3032 & 524 & 8 & - & 11 & 3298 & $: 8$ & 178 & 85 & 6 \\
\hline Fläsch & 230 & 186 & 59 & 37 & 5 & 5 & 319 & 10 & 1 & - & 1 & 325 & - & 2 & 4 & - \\
\hline Jenins & 173 & 133 & 136 & 83 & 6 & 1 & 377 & 17 & 1 & - & 3 & 391 & - & 5 & 2 & - \\
\hline Maienfeld & 658 & 474 & 380 & 364 & 86 & 16 & 1256 & 221 & 5 & - & 6 & 1398 & 6 & 46 & 32 & 6 \\
\hline Malans & 435 & 316 & 425 & 318 & 180 & 83 & 1080 & 276 & 1 & - & 1 & 1184 & 2 & 125 & 47 & - \\
\hline
\end{tabular}

Berufstätige nach Erwerbsklassen und Geschlecht

\begin{tabular}{|c|c|c|c|c|c|c|c|c|c|c|c|c|c|c|c|c|}
\hline & \multicolumn{16}{|c|}{ Berufstätige } \\
\hline & \multirow{2}{*}{\multicolumn{2}{|c|}{ Total }} & \multirow{2}{*}{\multicolumn{2}{|c|}{ Selbständige }} & \multicolumn{4}{|c|}{ Land- und Forstwirtschaft } & \multicolumn{4}{|c|}{ Industrie, Handwerk, Baugewerbe } & \multicolumn{4}{|c|}{ Handel, Verkehr, Gastgewerbe } \\
\hline & & & & & \multicolumn{2}{|c|}{ Total } & \multicolumn{2}{|c|}{ Sclbständige } & \multicolumn{2}{|c|}{ Total } & \multicolumn{2}{|c|}{ Selbständige } & \multicolumn{2}{|c|}{ Total } & \multicolumn{2}{|c|}{ Selbständige } \\
\hline & männlich & weiblich & | männlich $\mid$ & weiblich & männlich & weiblich & männlich & weiblich & männlich $\mid$ & weiblich & männlich & weiblith & männlich & weiblich & | männlich $\mid$ & weiblich \\
\hline Kreis Fünf Dörfer & 2585 & 683 & 419 & 48 & 488 & 63 & 236 & 19 & 1259 & 195 & 117 & 6 & 650 & 244 & 54 & 20 \\
\hline Haldenstein & 183 & 51 & 32 & 4 & 53 & 5 & 25 & 2 & 90 & 17 & 4 & 1 & 30 & 22 & 3 & 1 \\
\hline Igis & 1174 & 329 & 130 & 19 & 77 & 8 & 35 & $\overline{1}$ & 579 & 110 & 53 & 3 & 428 & 128 & 34 & 15 \\
\hline Mastrils & 131 & 18 & 32 & 2 & 37 & 1 & 25 & 1 & 49 & 8 & 3 & - & 37 & 5 & 4 & 1 \\
\hline Says & 38 & 6 & 15 & - & 27 & 4 & 14 & - & 8 & - & - & - & 2 & - & 1 & - \\
\hline Trimmis & 274 & 58 & 50 & 4 & 92 & 9 & 41 & 2 & 105 & 17 & 6 & 1 & 53 & 19 & 3 & - \\
\hline Untervaz & 400 & 90 & 87 & 10 & 116 & 23 & 54 & 6 & 221 & 20 & 29 & - & 42 & 33 & 3 & 3 \\
\hline Zizers & 385 & 131 & 73 & 9 & 86 & 13 & 42 & 7 & 207 & 23 & 22 & 1 & 58 & 37 & 6 & - \\
\hline Kreis Maienfeld & 1165 & 328 & 347 & 49 & 438 & 73 & 229 & 31 & 417 & 57 & 79 & 3 & 195 & 87 & 27 & 13 \\
\hline Fläsch & 119 & 23 & 49 & 4 & 68 & 8 & 46 & 3 & 33 & 3 & 3 & - & 12 & 5 & - & 1 \\
\hline Jenins & 124 & 41 & 52 & 13 & 67 & 21 & 42 & 12 & 33 & 5 & 4 & 1 & 18 & 6 & 6 & - \\
\hline Maienfeld & 489 & 145 & 144 & 21 & 163 & 22 & 83 & 11 & 180 & 17 & 43 & 1 & 73 & 42 & 12 & 8 \\
\hline Malans & 433 & 119 & 102 & 11 & 140 & 22 & 58 & 5 & 171 & 32 & 29 & 1 & 92 & 34 & 9 & 4 \\
\hline
\end{tabular}




\title{
EINIGE LITERATUR
}

1. Bernhard, Hans: Chur, Beitrag zur Siedlungs- und Wirtschaftsgeographie einer Verkehrsstadt. Chur 1937. 2. Bernhard, Hans: Ems, eine Bündner Gemeinde im wirtschaftlichen Aufstieg. Geographica Helvetica XVII 1962, Seite 296. 3. Brunner, Hans: Die Landschaft von Chur und Umgebung. Geographica Helvetica IV 1949, Seite 7o. 4. Durnwalder, Eugen: Der Weinbau des Bündner Rheintales, Wädenswil 1940. 5. Früh, J.: Geographie der Schweiz, Band III, Seite 499, St. Gallen 1938. 6. Gutersohn, Heinrich: Geographie der Schweiz, Band II, Seite 438, Bern 1961. 7. Kirchgraber, R.: Das Gebiet des ehemaligen Hochgerichtes Vier Dörfer, Zürich 1923. 8. Nigg, Werner: Die Landschaft im Unterricht der Mittelschule. Geographica Helvetica X 1955, Seite 162. 9. Nigg, Werner: Die Bündner Herrschaft. Geographica Helvetica XVII 1962, Seite 297. 10. Nıgg Werner: Die Büı:dner Herrschaft. "Schweizer Schulfunk»1965, Heft 5, Seite 163. 11. Schneider, Walter: Bekanntes und Neues von der Regionalplanung V Dörfer. «Terra Grischuna»1964, Nr. 5, Seite 247.

\section{TRANSFORMATIONS DU PAYSAGE \\ DANS LA VALLEE SEPTENTRIONALE DU RHIN (REGION COIRE)}

Le segment de vallée, long de 20 kilomètres forme une brèche large et profonde dans les montagnes du Nord des Grisons. Jusqu'à une date qui remonte à deux cents ans, le peuplement humain et les travaux agricoles s'étaient limités aux cônes de déjection, régions favorisées et protégées climatiquement, tandis que le fond de la vallée était ravagé périodiquement par les crues du Rhin.

Au cours du 19 e siècle, les habitants de la vallée ont corrigé le cours du fleuve au moyen de fortes digues. Depuis ce temps on peut exploiter le sol de la vallée. De nouvelles agglomérations (Landquart), des voies de communication et divers entreprises industrielles y ont pris naissance. Dans le district «Fünf Dörfer», la population a presque doublé en cent ans, tandis que dans les villages vignerons de la «Herrschaft», elle n'augmentait que de $17 \%$.

La vallée du Rhin (région de Coire, «Churer Rheintal»), sera encore plus industrialisée dans l'avenir et c'est une des tâches qui incombent à l'aménagement du territoire, que de prendre des mesures pour que cet espace vital, qui est sain et beau entre tous se développe au profit de la génération montante.

\section{ZUR ENTVÖLKERUNG DER GEMEINDE HEIMISWIL ZWISCHEN I9Io UND I960}

\author{
KLAUS AERNI
}

Die bernische Mittellandgemeinde Heimiswil liegt am Rande des niederen Berglandes, das nördlich der Eisenbahnlinie Langenthal-Herzogenbuchsee-Burgdorf-Bern an das Hügelland grenzt (Fig. 1, LK 1: 25 000, Nr. 1147 und 1148). Der Untergrund besteht aus Gesteinen der oberen Meeresmolasse, im Norden überlagert durch Würmmoränen des Rhonegletschers, im Haupttal überdeckt durch Niederterrassenschotter (1).

Es ergibt sich demnach folgende morphologische Gliederung (Fig. 2):

Im Talboden finden wir eine weite, leicht durchtalte Terrassenfläche, die sich mit $2 \%$ Gefälle gegen den Emmelauf neigt. Dies ist der würmeiszeitliche Talboden, der sich vom Gebiet der Ziegelei über Ried, Stöckeren, Chipf, Dörfli, Schüren, Garnöu bis in die Dreienmatt verfolgen läßt, im oberen Teil jedoch etwas steiler wird. Im unteren Talabschnitt ist der Boden lehmig und schwer, teilweise mit Sand und Geröll vermischt. Die Namen Blaumatt und Ried und vor allem die Gruben der Ziegelei verraten das Vorkommen von Staulehmen. Heute ist das Gebiet drainiert; aber noch vor zwei Generationen gab es hier Riedmatten mit Schilf.

Einen anderen Charakter zeigt das Gebiet zwischen der Egg und dem Steilabfall ins Tal von Grafenschüren-Bickigen und dem Chänerechgraben: Die Hochfläche von Hueb-Guetisberg ist 